\title{
Domain Identification for Inverse Problem via Conformal Mapping and Fixed Point Methods in Two Dimensions
}

\author{
Fagueye Ndiaye \\ Université Cheikh Anta Diop de Dakar, Faculté des Sciences et Technologies de l'Éducation et de la Formation, \\ BP 5036 Dakar-Fann, Senegal
}

Correspondence should be addressed to Fagueye Ndiaye; fagueye.ndiaye@ucad.edu.sn

Received 25 April 2020; Accepted 17 June 2020; Published 25 August 2020

Academic Editor: Fasma Diele

Copyright (C) 2020 Fagueye Ndiaye. This is an open access article distributed under the Creative Commons Attribution License, which permits unrestricted use, distribution, and reproduction in any medium, provided the original work is properly cited.

In this paper, we present a survey of the inverse eigenvalue problem for a Laplacian equation based on available Cauchy data on a known part $\Gamma_{0}$ and a homogeneous Dirichlet condition on an unknown part $\Gamma_{0}$ of the boundary of a bounded domain, $\Omega \subset \mathbb{R}^{N}$. We consider variations in the eigenvalues and propose a conformal mapping tool to reconstruct a part of the boundary curve of the twodimensional bounded domain based on the Cauchy data of a holomorphic function that maps the unit disk onto the unknown domain. The boundary values of this holomorphic function are obtained by solving a nonlocal differential Bessel equation. Then, the unknown boundary is obtained as the image of the boundary of the unit disk by solving an ill-posed Cauchy problem for holomorphic functions via a regularized power expansion. The Cauchy data were restricted to a nonvanishing function and to the normal derivatives without zeros. We prove the existence and uniqueness of the holomorphic function being considered and use the fixed-point method to numerically analyze the results of convergence. We'll calculate the eigenvalues and compare the result with the shape obtained via minimization functional method, as developed in a previous study. Further, we'll observe via simulations the shape of $\Gamma$ and if it preserves its properties with varying the eigenvalues.

\section{Introduction}

In the paper, we consider the mathematical modeling employed in electrostatic imaging during nondestructive testing. This analysis engenders an inverse boundary value problem for a Laplace equation. This problem entails the identification of an unknown domain, $\Gamma$, within a conducting host medium with constant conductivity based on known Cauchy data on the boundary of the medium, $\Gamma_{0}$. For detailed examples, please consult [1].

We suppose that $\Omega$ is a simply connected domain in $\mathbb{R}^{N}$ with $C^{2}$ smooth boundaries $\partial \Omega$ and

$$
\partial \Omega=\Gamma_{0} \mathrm{o} \Gamma
$$

The boundary $\Gamma$ is assessed by imposing a voltage pattern at a number of electrodes attached to the boundary $\Gamma_{0}$ and measuring the resultant current through the electrodes.
The inverse problem we are concerned with is to determine the unknown part, $\Gamma$, of the boundary of the bounded domain $\Omega \subset \mathbb{R}^{N}$, based on available Cauchy data $(g, h)$ on $\Gamma_{0}$ :

$$
\left\{\begin{array}{l}
u_{\Omega}=g \text { on } \Gamma_{0}, \\
\frac{\partial u}{\partial v}=h \text { on } \Gamma_{0},
\end{array}\right.
$$

where $u \in H^{1}(\Omega)$ denotes a solution of the Laplace equation:

$$
-\Delta u_{\Omega}=\lambda_{\Omega} u_{\Omega} \text { in } \Omega, \lambda_{\Omega} \text { eigenvalue, }
$$

satisfying the condition:

$$
u_{\Omega}=0 \text { on } \Gamma \text {. }
$$

where $g$ denotes a continuous positive function on $\Gamma_{0}, h$ 
denotes a continuous negative function on $\Gamma_{0}$, and $v$ denotes the outer normal vector unit.

My thesis (please consult [2]) is aimed at identifying $\Gamma$ via Cauchy data on $\Gamma_{0}$ using methods based on conformal mapping and the minimization function. The results (please consult [3]) were published in AMO (Advanced Modeling and Optimization) in 2014.

Here, we aim to compare the results obtained using conformal mapping and the fixed-point method and observe the variations in the shape of $\Gamma$ with varying eigenvalues.

\section{Methods}

2.1. Conformal Mapping Method. The conformal mapping method was presented in [3].

This paper is primarily aimed at presenting iterative methods to solve the inverse problem $(1)-(2)-(3)$ by using ideas from conformal mapping theory that have been developed over the last decade. For detailed references, please consult $[1,4,5]$.

However, in our approach, we consider a spectral problem on the simply connected domain $\Omega$ in $\mathbb{R}^{N}$ with $C^{2}$ smooth boundary $\partial \Omega$ satisfying (1).

The family of holomorphic functions corresponding to the eigenvalues of the spectrum was identified via the fixedpoint method and Nyström method.

The proposed reconstruction of the boundary curve $\Gamma$ based on available Cauchy data on the accessible boundary, $\Gamma_{0}$, is based on a conformal map $\Phi: D_{1} \rightarrow \Omega$. Thus, we consider $\Gamma_{D_{1}}$, the boundary of the complex disk $D_{1}$ with radius one centered at the origin, and map $\Gamma_{D_{1}}$ onto $\partial \Omega$ via $\Phi$. We introduce the following notations.

$$
\begin{aligned}
& \Gamma_{2}=\left\{e^{i \theta}, 0 \leq \theta \leq \alpha \pi, 0<\alpha<2\right\}, \\
& \Gamma_{1}=\left\{e^{i \theta}, \alpha \pi<\theta \leq 2 \pi, 0<\alpha<2\right\} .
\end{aligned}
$$

Further, $\Gamma$ and $\Gamma_{0}$ are parameterized as follows:

$$
\begin{gathered}
\Gamma_{0}=\{\Psi(\theta), \theta \in[0, \alpha \pi]\}, \\
\Gamma=\{\Psi(\theta), \theta \in] \alpha \pi, 2 \pi]\},
\end{gathered}
$$

where $\Psi: \mathbb{R} \rightarrow \mathbb{C}$ is a continuous differentiable $2 \pi$ - periodic from $\mathbb{R}$ onto $\mathbb{C}$ satisfying the property that $\left.\Psi\right|_{[0,2 \pi]}$ is injective. This property implies that

$$
\left|\Psi^{\prime}(\theta)\right| \neq 0, \forall \theta \in[0,2 \pi]
$$

We can normalize the mapping $\Phi$ by prescribing $\Phi(1)=\Psi(0)$ and define the bijective boundary correspondence function $\Psi:[0,2 \pi] \longrightarrow \mathbb{C}$ by setting

$$
\Psi(\theta):=\Phi\left(e^{i \theta}\right), \theta \in[0,2 \pi]
$$

It is clear that the values of $\Phi$ on the boundary produce a unique solution $\Psi$ of the Cauchy problem, given by

$$
\Phi\left(e^{i \theta}\right)=\Psi(\theta)
$$

Therefore, the operator $\Psi(\theta)=\Phi\left(e^{i \theta}\right), \theta \in[0,2 \pi]$ is well defined.

The Cauchy-Riemann equations give rise to nonlinear ordinary differential equations via Bessel equations for the values on this part of the boundary $\left.\Psi\right|_{\Gamma_{2}}$

$$
\Phi^{\prime}\left(e^{i \theta}\right)=\frac{\partial \widehat{u} / \partial v}{h o \Phi\left(e^{i \theta}\right)} \text { on } \Gamma_{2}
$$

which is to be determined via the fixed-point method.

(10) is followed by the solution of several ill-posed Cauchy problems with respect to the holomorphic function $\Phi$ by using a regularized power series expansion to retrieve the unknown part of the boundary curve using $\Phi\left(\Gamma_{1}\right)=\Gamma$.

2.2. Position of Problem. The inverse problem (1) - (2) - (3) is thereby reduced to the determination of a holomorphic function $\Phi$ that is a solution of

$$
\left\{\begin{array}{l}
(i) \Phi\left(\Gamma_{2}\right)=\Gamma, \\
(i i) \widehat{u}=\widehat{g} \text { on } \Gamma_{1}, \\
(i i i) \widehat{u}=0 \text { on } \Gamma_{2}, \\
(i v)-\Delta \widehat{u}=\widehat{\lambda} \widehat{u} \text { in } D_{1} \text {, with } \widehat{\lambda}=\lambda\left|\Phi^{\prime}(z)\right|^{2}, \\
(v) \frac{\partial}{\partial v} \widehat{u}\left(e^{i \theta}\right)=h\left(\Phi\left(e^{i \theta}\right)\right) \Phi^{\prime}\left(e^{i \theta}\right) \text { on } \Gamma_{2} .
\end{array}\right.
$$

with $\widehat{u} \in H^{1}\left(D_{1}\right)$.

Using the polar coordinates of the solution $\widehat{u}$ of the Dirichlet problem on the disk $D_{1}$ with boundary conditions and the solution of the Bessel equation of order $k$, see [3], we have

$$
\widehat{u}_{n}(r, \theta)=\sum_{k=0}^{+\infty} A_{k, n} J_{k}\left(r l_{k, n}\right) e^{i k \theta},
$$

with

$$
A_{k, n}^{2} \approx \frac{8 \lambda_{k, n}}{\pi\left(4 \lambda_{k, n}-\left(1+(-1)^{k}\right)\right)}, \forall k \geq 0, n \geq 1
$$

where $J_{k}$ denotes the Bessel function of order $k$ and $\lambda_{k, n}$ corresponds to $\widehat{u}_{k, n}$.

Therefore, by

$$
\frac{\partial}{\partial v} \widehat{u}\left(e^{i \theta}\right)=h\left(\Phi\left(e^{i \theta}\right)\right) \Phi^{\prime}\left(e^{i \theta}\right) \text { on } \Gamma_{2}
$$


we determine a family of holomorphic functions, $\Phi_{n}$, such that

$$
\Phi_{n}^{\prime}\left(e^{i \theta}\right) \approx \frac{\sum_{k=0}^{+\infty} p_{k, n}\left(\lambda_{k, n}\right) e^{i k \theta}}{h o \Phi_{n}\left(e^{i \theta}\right)}, \forall n \geq 1
$$

with

$$
p_{k, n}(x)=\frac{2 x(-1)^{n} \sqrt{2 / \pi}}{\sqrt{x-(1 / 2)\left(1+(-1)^{k}\right)}}, \quad x \geq \frac{\pi}{4}
$$

$p$ is well defined. We present the details of this algorithm, including a convergence analysis result in the following sections.

\section{Results and Discussion}

3.1. Presentation of the Fixed Point Method. A fixed point is a solution of equation $T x=x$ where $T$ is an operator defined from a nonempty set $M$ onto itself. The principal condition for a nonlinear operator to admit a fixed point is for it to be a strict contraction.

The determination of $\Phi(z)$ is based on the Banach fixedpoint theorem, where a contracting nonlinear application on a complete metric space admits a fixed point [1].

General methods of construction of approximate conformal maps can be found in survey works by $[1,5,6]$.

3.1.1. Nonlocal Differential Equation. In this section, we attempt to determinate $\Phi$, satisfying (15), using the fixed point method.

Let $A: H^{1 / 2}[0,2 \pi] \times H^{1 / 2}[0,2 \pi] \longrightarrow H^{-1 / 2}[0,2 \pi]$ denote the Dirichlet-Neumann operator on $\Gamma_{D_{1}}$, which maps $\left(\widehat{u}_{1}, \widehat{u}_{2}\right)$ onto the normal derivative of $\widehat{u} \in H^{1}\left(D_{1}\right)$, defined as follows:

$$
A\left(\widehat{u}_{1}, \widehat{u}_{2}\right):=\frac{\partial \widehat{u}}{\partial v}\left(e^{i \theta}\right), \theta \in[0,2 \pi]
$$

with the respective boundary values given by

$$
\left\{\begin{array}{l}
\widehat{u}\left(e^{i \theta}\right)=\widehat{u}_{1} \text { on } \Gamma_{1}, \\
\widehat{u}\left(e^{i \theta}\right)=\widehat{u}_{2} \text { on } \Gamma_{2} .
\end{array}\right.
$$

The boundary traces on $\Gamma_{1}$ and $\Gamma_{2}$ are, respectively, given by

$$
\left\{\begin{array}{l}
\widehat{u}\left(e^{i \theta}\right)=\widehat{g} \text { on } \Gamma_{1}, \\
\widehat{u}\left(e^{i \theta}\right)=0 \text { on } \Gamma_{2} \\
\frac{\partial \widehat{u}}{\partial v}\left(e^{i \theta}\right)=\Phi_{n}^{\prime}\left(e^{i \theta}\right) h\left(\Phi_{n}\left(e^{i \theta}\right)\right) \text { on } \Gamma_{2} .
\end{array}\right.
$$

However, we have the nonlocal differential equation for the conformal map $\Phi_{n}$, which is a critical element in the inverse algorithm.

$$
\Phi_{n}^{\prime}\left(e^{i \theta}\right)=\frac{A\left(\widehat{u}_{1}, \widehat{u}_{2}\right)}{h\left(\Phi_{n}\left(e^{i \theta}\right)\right)}
$$

The boundary correspondence function, $\Psi_{n}$, is a solution of

$$
\Psi_{n}^{\prime}(\theta)=\frac{A\left(u_{1}\left(\Psi_{n}(\theta)\right), u_{2}\left(\Psi_{n}(\theta)\right)\right)}{h\left(\Psi_{n}(\theta)\right)}
$$

As $h<0$, equations (20) and (21) are well defined.

Based on the available Cauchy data, $(g, h)$, we have (12)

$$
\widehat{u}_{n}(r, \theta) \approx \sum_{k=0}^{+\infty} \frac{\sqrt{2 \pi}(2 n+k-1) \cos (k(\pi / 2)-r(\pi / 2)(2 n+k-1)) e^{i k \theta}}{\sqrt{\pi^{2}(2 n+k-1)^{2}-2\left(1+(-1)^{k}\right)}}, n \geq 1 .
$$

the fundamental solution of (11)

Let us discuss some definitions and properties related to fixed points before employing the fixed-point methods to determinate $\Phi$.

\subsubsection{Fixed-Point Theorems (See [1])}

Theorem 1. (Fixed-Point theorem of Banach)
Let $f: S \longrightarrow S$ be a nonlinear operator where $S$ is a complete metric space. We assume that $f$ is a strict contraction. Then, $f$ admits a unique fixed point (see [7]).

Proof of Theorem 2. Consider any point $x_{0} \in S$ and iteratively define

$$
x_{n+1}=f\left(x_{n}\right) \text { for } n=0,1, \cdots \text {. }
$$


Then,

$$
\begin{gathered}
\left\|f\left(x_{n+1}\right)-f\left(x_{n}\right)\right\| \leq \gamma\left\|x_{n+1}-x_{n}\right\|=\gamma\left\|f\left(x_{n}\right)-f\left(x_{n-1}\right)\right\|, \\
\left\|f\left(x_{n+1}\right)-f\left(x_{n}\right)\right\| \leq \gamma^{n+1}\left\|f\left(x_{0}\right)-x_{0}\right\| \text { pour } n=0,2, \cdots .
\end{gathered}
$$

If $n \geq k$, then

$$
\begin{aligned}
\left\|x_{n}-x_{k}\right\| & =\left\|f\left(x_{n-1}\right)-f\left(x_{k-1}\right)\right\|, \\
& \leq \sum_{j=k-1}^{n-2}\left\|f\left(x_{j+1}\right)-f\left(x_{j}\right)\right\|, \\
& \leq\left\|f\left(x_{0}\right)-x_{0}\right\| \sum_{j=k}^{n-1} \gamma^{j} .
\end{aligned}
$$

Hence $\left\{x_{k}\right\}_{k=1}^{\infty}$ is a Cauchy sequence in $S$, and, as $S$ is complete, there exists $x \in S$ such that $x_{k} \longrightarrow x$ in $S$. Thus, it is evident that

$$
f(x)=x .
$$

Hence, $x$ is fixed point for $f$ and the fact that $f$ is a strict contraction ensures its uniqueness.

The following is the version of the Banach fixed-point theorem and its corollary that is used in the subsequent proofs.

Theorem 3. Let $(S, d)$ be a nonempty complete metric space. If $f: S \longrightarrow S$ is such that, for at least one integer $m \geq 1, f^{m}$ is a strict contraction, then $f$ admits an unique fixed point.

Theorem 4. Let $(X, d)$ be a complete metric space and let $T: X \longrightarrow X$ be a $(\delta, L)$-almost contraction. Then,

$$
\operatorname{Fix}(T)=\{x \in X, T x=x\} \neq \varnothing .
$$

(1) For any $x_{0} \in X$, the Picard iteration $\left\{x_{m}\right\}_{m=0}^{\infty}$, $x_{m}=T^{m} x_{0}$ converges to some $x^{*} \in \operatorname{Fix}(T)$;

(2) The following estimate holds

$d\left(x_{m+i-1}, x^{*}\right) \leq \frac{\delta^{i}}{1-\delta} d\left(x_{m}, x_{m-1}\right), \quad m=0,1,2, \cdots, \quad i=1,2 . \cdots$

3.1.3. Fixed-Point Equation and Iteration. Let $S$ be an operator defined as follows:

$$
S \psi(\theta)=\theta+\psi(\theta)
$$

$$
\text { with } \psi: H^{1 / 2}[0, \alpha \pi] \longrightarrow H^{1 / 2}[0, \alpha \pi] \text {. }
$$

We assume that $\psi$ is not identically zero.
Further, we introduce an operator $T: H_{0}^{1}[0, \alpha \pi] \longrightarrow$ $H_{0}^{1}[0, \alpha \pi], 0<\alpha<2$ defined by

$$
(T \psi)(\theta):=\frac{\alpha}{2} \int_{0}^{\theta}\left(U \psi-\frac{1}{\alpha \pi} \int_{0}^{\alpha \pi} U \psi d \theta\right) d \tau, \theta \in[0, \alpha \pi],
$$

with

$$
U \psi:=\frac{A\left(u_{1} o S \psi, u_{2} o S \psi\right)}{h o S \psi}
$$

By integrating (32) and considering that $\psi(0)=0$ and $\psi(\alpha \pi)=0$, we obtain

$$
\frac{1}{\alpha \pi} \int_{0}^{\alpha \pi} \frac{A\left(u_{1} o S \psi, u_{2} o S \psi\right)}{h o S \psi}=1 .
$$

Using the boundary conditions of $\psi$, we can express $\psi(\theta)=b\left(e^{i \theta^{2}(\alpha \pi-\theta)^{2}}-1\right), b \in \mathbb{C}$.

It is easy to verify that $T$ is nonlinear and contracting.

Now, we use the following theorem (see [1]).

Theorem 5. Let $(\kappa, \tau)$ be a pair of Cauchy data obtained from (8).

Then, in terms of the holomorphic map $\Phi_{n}: D_{1} \longrightarrow \Omega$ and its boundary correspondence function $\Psi_{n}$, the function $\psi_{n}=S^{-1} \Psi_{n}$ is a fixed point for $T$.

We obtain a fixed point defined by successive iteration of $T$ beginning with an arbitrary approximation $\psi_{n_{0}}$, for all $n \geq 1$. Therefore, Theorem 3.4 induces the following iteration:

$$
\psi_{n_{m+1}}=T \psi_{n_{m}}, \quad m=0,1,2, \cdots .
$$

The solution of the Cauchy problem is required to identify the boundary function $\Psi_{n}$ from the function $\psi_{n}$. For this purpose, let us express $S \psi_{n}$ using the following expansion:

$$
S \psi_{n}(\theta)=\sum_{k=0}^{+\infty} a_{n, k} e^{i k \theta}, \theta \in[0, \alpha \pi] .
$$

Using $\Phi_{n}\left(e^{i \theta}\right)=S \psi_{n}(\theta)$, we obtain $\Phi_{n}$ using the following expansion:

$$
\Phi_{n}(z)=\sum_{k=0}^{+\infty} a_{n, k} e^{i k \theta}, \theta \in[0, \alpha \pi]
$$

The following convergence result justifies the procedure of iteration (31).

Theorem 6 (see [1]). Let $D_{1}$ be the unit disk and $g$ be a continuous positive function defined on it. Then, successive approximations

$$
\psi_{n_{m+1}}=T \psi_{n_{m}}, m=0,1,2, \cdots,
$$


converge provided that the initial estimate $\Psi_{n_{0}}$ of the boundary function is sufficiently close to the exact function $\psi_{n}$.

Proof (see [4]). Theorem 6 is proved by first establishing that $\left\|T^{\prime}(0)\right\| \leq 1$ in the case where $\Omega=D_{1}$. Thereafter, a continuity argument is applied to extend the estimate to the norm of $T^{\prime}\left(\psi_{n}\right)$ for $\psi_{n}$ in a neighborhood of the fixed point defined in Theorem 3.4.

Proof of Theorem 7. We calculate the derivate of the nonlinear operator $T$. We begin by estimating $T^{\prime}(0)$. We have

$$
\left\|T^{\prime}(0)\right\|=0 \text {. }
$$

Therefore, we have, $\left\|T^{\prime}(0)\right\|<1, \quad \forall 0<\alpha<2$.

Using a continuity argument to estimate the norm of $T^{\prime}$ $\psi_{n}(\theta)$ near the fixed point of Theorem 3.4, we have for all $\theta$ $\in[0, \alpha \pi]$,

$$
\left\|T^{\prime} \psi_{n}(\theta)\right\|=\frac{\alpha}{2}\left\|\left(\psi_{n}\right)^{\prime}(\theta)\right\|
$$

Thus, $\left\|T^{\prime} \psi_{n}(\theta)\right\|<\left\|\left(\psi_{n}\right)^{\prime}(\theta)\right\|, \quad \forall \theta \in[0, \alpha \pi]$ and $n \geq 1$.

The approximation of $T^{\prime} \psi_{n}(\theta)$ near the fixed point of the theorem is $\left(\psi_{n}\right)^{\prime}(\theta), n \geq 1$.

We now determinate a numerical approximation of the conformal map $\Phi_{n, k}$, for all $n \geq 1$.

3.1.4. Numerical Approximation of the Conformal Map. In this section, we use an ordinary differential equation of the boundary correspondence function to obtain an approximation of the conformal map, $\Phi_{\mathrm{n}}$, for all $n \geq 1$.

Let us introduce two boundary correspondence functions, $\Psi_{n}^{2}$ and $\Psi_{\mathrm{n}}^{1}$, defined from $[0,2 \pi]$ to $\mathbb{C} b y$

$$
\left\{\begin{array}{l}
\Psi_{n}^{1}(\theta):=\Phi_{n}\left(e^{i \theta}\right) \text { pour } \theta \in[0, \alpha \pi] \\
\Psi_{n}^{2}(\theta):=\Phi_{n}\left(e^{i \theta}\right) \text { pour } \theta \in[\alpha \pi, 2 \pi]
\end{array}\right.
$$

On $\Gamma_{2}$, equation (21) becomes

$$
\left(\Psi_{n}^{1}\right)^{\prime}(\theta)=\frac{\partial \widehat{u} / \partial r}{h\left(\Psi_{n}^{1}(\theta)\right)}
$$

Here, we use the length of $\operatorname{arc} \Gamma_{2}$ determined by $d \theta$. By removing the identity,

$$
\frac{1}{\alpha \pi} \int_{0}^{\alpha \pi} \frac{\partial \widehat{u} / \partial r}{h\left(\Psi_{n}^{1}(\theta)\right)} d \theta=1
$$

that is obtained by integrating (41). Then, we have for $\psi_{n}^{1}(\theta):=\Psi_{n}^{1}(\theta)-\theta$, the equation

$$
\left(\psi_{n}^{1}\right)^{\prime}(\theta)=U \psi_{n}^{1}(\theta)-\frac{1}{\alpha \pi} \int_{0}^{\alpha \pi} U \psi_{n}^{1} d \theta
$$

where we have for convenience

$$
U\left(\psi_{n}^{1}\right)=\frac{\partial \widehat{u} / \partial r}{h o S \psi_{n}^{1}}
$$

with $S$ operator defined in (35).

For

$$
\psi_{n}^{1}(\theta):=\int_{0}^{\theta}\left(U \psi_{n}^{1}-\frac{1}{\alpha \pi} \int_{0}^{\alpha \pi} U \psi_{n}^{1} d \theta\right) d \tau, \theta \in[0, \alpha \pi],
$$

the iterative solution of (43), we have the following convergence results:

Theorem 8. The successive approximations defined by (45) converge from the initial approximation $\psi_{n_{0}}^{1}=\theta(\alpha \pi-\theta)$ provided that $\Omega$ is close enough to $D_{1}$.

When we obtain $\psi_{n}^{1}$, that is, $\Phi_{n}$, the boundary values of $\Phi_{n}$ on $\Gamma_{2}$ to be used for the iteration of (45) are given by

$$
\Phi_{n}\left(e^{i \theta}\right)=\sum_{k=0}^{+\infty} a_{n, k} e^{i k \theta}
$$

with

$$
\begin{aligned}
& 2 a_{n, k} \pi=\int_{0}^{2 \pi} \Phi_{n}\left(e^{i \theta}\right) e^{-i k \theta} d \theta, \\
& 2 a_{n, k} i \pi=\int_{\Gamma_{D_{1}}}(\bar{z})^{k+1} \Phi_{n}(z) d z,
\end{aligned}
$$

So, on $\Gamma_{2}$,

$$
a_{n, k} \alpha \pi=\int_{\Gamma_{2}} \Phi_{n}\left(e^{i \theta}\right) e^{-i k \theta} d \theta
$$

We can express the expansion of $\Phi_{n}$ in the form

$$
\Phi_{n}(z)=\sum_{k=0}^{+\infty} a_{n, k} z^{k}
$$

which is numerically stable for $|z|<1$. By construction, considering (40), we have $\Phi_{n}(1)=\Psi_{n}^{1}(0)$ and $\Phi_{n}\left(e^{i \alpha \pi}\right)=\Psi_{n}^{1}(\alpha \pi)$.

Now, we describe the numerical algorithm that we will implement in this paper. We use a method involving integral equations on $\Gamma_{2}$. The integral equation obtained is resolved via the Nyström method.

We give ourselves a regular mesh,

$$
\theta_{m}=\theta_{0}+m h, 1 \leq m \leq N, h=\frac{\alpha \pi}{N} \text { on }[0, \alpha \pi] .
$$

The approximations $\Psi_{n_{m}}^{1}$ of $\Psi_{n}^{1}\left(\theta_{m}\right)$ at the nodes of mesh are calculated using the q previous approximate values $y_{m-1}$, $y_{m-2}, \cdots, y_{m-q}$. 
The iterations allow approximations of the normal derivatives via trigonometric interpolation.

The integration interpolation polynomial on $\left[0, \theta_{m}\right]$ is used to associate $\Psi_{n_{m}}^{1}$ to $\Psi_{n_{m+1}}^{1}$, via (43), with $m=1, \cdots, N$.

Beginning with identity, we have

$$
\psi_{n}^{1}(\theta+t)=\psi_{n}^{1}(\theta)+\int_{\theta}^{\theta+t}\left(U \psi_{n}^{1}(\tau)-\frac{1}{\alpha \pi} \int_{0}^{\alpha \pi} U \psi_{n}^{1} d \xi\right) d \tau
$$

which corresponds to

$$
\psi_{n_{m+1}}^{1}=\psi_{n_{m}}^{1}+\int_{\theta_{m}}^{\theta_{m+1}}\left(U \psi_{n}^{1}(\tau)-1\right) d \tau
$$

or more precisely

$$
\psi_{n_{m+1}}^{1}=\psi_{n_{m}}^{1}+\int_{\theta_{m}}^{\theta_{m+1}}\left[\frac{\sum_{k=0}^{+\infty} p_{k, n}\left(\lambda_{k, n}\right) e^{i k \tau}}{h o S \psi_{n}^{1}(\tau)}-1\right] d \tau
$$

We substitute $\left[\left(\left(\sum_{k=0}^{+\infty} p_{k, n}\left(\lambda_{k, n}\right) e^{i k \tau}\right) /\left(h o S \psi_{n}^{1}(\tau)\right)\right)-1\right]$ in the integral via the Nyström method using the interpolation polynomial $P(\theta)$.

3.1.5. Nyström Method (See [8]). This method, called the quadrature method, is the application of numerical methods of calculating integrals to obtain a linear system. We write

$$
\psi_{n}^{1}\left(\theta_{m+1}\right)=\psi_{n}^{1}\left(\theta_{m-1}\right)+\int_{\theta_{m-1}}^{\theta_{m+1}} P(\theta) d \theta
$$

where $P$ denotes the interpolation polynomial of Lagrange of degree $q-1$, for the pair of values

$$
\left(\theta_{m-j}, f_{m-j}\right), 0 \leq j \leq q-1
$$

where we use

$$
\begin{gathered}
U \psi_{n j}^{1}=U \psi_{n}^{1}\left(\theta_{j}\right), \\
f_{m-j}=f\left(\theta_{m-j}\right)=\left(\frac{\sum_{k=0}^{+\infty} p_{k, n}\left(\lambda_{k, n}\right) e^{i k \theta_{m-j}}}{h o S \psi_{n}^{1}\left(\theta_{m-j}\right)}-1\right), \\
P(\theta)=\sum_{j=0}^{q-1} f\left(\theta_{m-j}\right) l_{m-j}(\theta),
\end{gathered}
$$

with the polynomials $l_{m-j}(\theta)$ are Lagrange polynomials of $\mathbb{R}_{n}[X]$ associated to $\theta_{m}, \theta_{m-1}, \theta_{m-2}, \cdots, \theta_{m-q+1}$. That is,

$$
\psi_{n_{m+1}}^{1}=\psi_{n_{m-1}}^{1}+\frac{\alpha \pi}{N} \sum_{j=0}^{q-1} \gamma_{q, j} f_{m-j}, N \in \mathbb{N}^{*}
$$

Explicitly, we can express (57) in the form

$\psi_{n_{m+1}}^{1}=\psi_{n_{m-1}}^{1}+\frac{\alpha \pi}{N} \sum_{j=0}^{q-1} \gamma_{q, j}\left(\frac{\sum_{k=0}^{+\infty} p_{k, n}\left(\lambda_{k, n}\right) e^{i k \theta_{m-j}}}{h o S \psi_{n}^{1}\left(\theta_{m-j}\right)}-1\right), N \in \mathbb{N}^{*}$.

Therefore, we can set $q=2,3, \ldots$ for the approximations. Hence,

$$
\text { If } \begin{aligned}
q & =2 \text { then } \psi_{n_{m+1}}^{1} \\
& =\psi_{n_{m-1}}^{1}+\frac{2 \alpha \pi}{N}\left(\frac{\sum_{k=0}^{+\infty} p_{k, n}\left(\lambda_{k, n}\right) e^{i k \theta_{m}}}{h o S \psi_{n}^{1}\left(\theta_{m}\right)}-1\right) N \in \mathbb{N}^{*} .
\end{aligned}
$$

$$
\text { If } q=3 \text { then }
$$

$$
\begin{aligned}
\psi_{n_{m+1}}^{1}= & \psi_{n_{m-1}}^{1}+\frac{\alpha \pi}{3 N}\left(7 \frac{\sum_{k=0}^{+\infty} p_{k, n}\left(\lambda_{k, n}\right) e^{i k \theta_{m}}}{h o S \psi_{n}^{1}\left(\theta_{m}\right)}\right. \\
& \left.-2 \frac{\sum_{k=0}^{+\infty} p_{k, n}\left(\lambda_{k, n}\right) e^{i k \theta_{m-1}}}{h o S \psi_{n}^{1}\left(\theta_{m-1}\right)}+\frac{\sum_{k=0}^{+\infty} p_{k, n}\left(\lambda_{k, n}\right) e^{i k \theta_{m-2}}}{h o S \psi_{n}^{1}\left(\theta_{m-2}\right)}-6\right) .
\end{aligned}
$$

If $q=4$, then

$$
\begin{aligned}
\psi_{n_{m+1}}^{1}= & \psi_{n_{m-1}}^{1}+\frac{\alpha \pi}{3 N}\left(8 \frac{\sum_{k=0}^{+\infty} p_{k, n}\left(\lambda_{k, n}\right) e^{i k \theta_{m}}}{h o S \psi_{n}^{1}\left(\theta_{m}\right)}-5 \frac{\sum_{k=0}^{+\infty} p_{k, n}\left(\lambda_{k, n}\right) e^{i k \theta_{m-1}}}{h o S \psi_{n}^{1}\left(\theta_{m-1}\right)}\right. \\
& \left.+4 \frac{\sum_{k=0}^{+\infty} p_{k, n}\left(\lambda_{k, n}\right) e^{i k \theta_{m-2}}}{h o S \psi_{n}^{1}\left(\theta_{m-2}\right)}-\frac{\sum_{k=0}^{+\infty} p_{k, n}\left(\lambda_{k, n}\right) e^{i k \theta_{m-3}}}{h o S \psi_{n}^{1}\left(\theta_{m-3}\right)}-6\right)
\end{aligned}
$$

After fixing $\psi_{n_{0}}^{1}$, we calculate $\psi_{n_{1}}^{1}$ using equation (45), for all $q$.

The estimate of $\psi_{n}^{1}$ allows us to calculate $a_{n, k}, k=0,1, \cdots, J$ using (29), (35), and (48) and, subsequently, the expression of $\Phi_{n_{I}}^{1}, \quad J \in \mathbb{N}$.

We use

$$
a_{n, k}=\alpha_{n, k}+i \beta_{n, k} \text { with } 0 \leq k \leq J
$$

The traces of the curves corresponding to the equations defined below provide an approximate domain.

$$
\begin{aligned}
& \mathfrak{R}\left(\Phi_{n_{J}}^{1}\right)=\sum_{k=0}^{J} \alpha_{n, k} \cos k \theta-\beta_{n, k} \sin k \theta, \\
& \mathfrak{J}\left(\Phi_{n_{J}}^{1}\right)=\sum_{k=0}^{J} \alpha_{n, k} \sin k \theta+\beta_{n, k} \cos k \theta .
\end{aligned}
$$

We attempt to determine $\psi_{n}^{1}$ for $q=2$. The approach for the other case is similar. 
We know that (59) is equivalent to

$$
\psi_{n_{m+1}}^{1}=\psi_{n_{m-1}}^{1}+\frac{2 \alpha \pi}{N}\left(\psi_{n_{m}}^{1}\right)^{\prime}, \quad N \in \mathbb{N}^{*}, \text { for } q=2
$$

After calculation, we have

$$
\psi_{n_{m+1}}^{1}=\psi_{n_{0}}^{1}+\frac{2 \alpha \pi}{N}\left(\sum_{j=0}^{1 / 2(m-1)} \psi_{n_{2 j+1}}^{1^{\prime}}\right)
$$

or

$$
\psi_{n_{m+1}}^{1}=\psi_{n_{0}}^{1}+\frac{4 \pi}{N}\left(\sum_{j=0}^{1 / 2(m-1)} T \psi_{n_{2 j+1}}^{1}\right)^{\prime}, \quad N \in \mathbb{N}^{*} .
$$

3.1.6. Convergence Results of $\left(\psi_{n_{m}}^{1}\right)_{m}$. We set $U_{m+1}=\psi_{n_{m+1}}^{1}$, as defined above.

For $\boldsymbol{q}=\mathbf{2}$, we have $\left(\boldsymbol{U}_{\boldsymbol{m}}\right)_{\boldsymbol{m} \geq \mathbf{0}}$ a sequence defined in $[0, \boldsymbol{\alpha}], 0<\boldsymbol{\alpha}<2$, by

$$
U_{m+1}=U_{m-1}+\frac{4 \pi}{N}\left(T U_{m}\right)^{\prime}, \quad N \in \mathbb{N}^{*} .
$$

The Picard theorem ensures the convergence of $\left.\left(U_{m}\right)_{m \geq 0}\right)$.

We can write

$$
\begin{aligned}
U_{m+1} & =U_{0}+\frac{4 \pi}{N}\left(T U_{1}+T U_{3}+T U_{5}+\cdots+T U_{m}\right)^{\prime}, \\
& =U_{0}+\frac{4 \pi}{N}\left(T U_{1} \frac{1-(\alpha / 2)^{4 m-4}}{1-(\alpha / 2)^{2}}\right), \quad N \in \mathbb{N}^{*} .
\end{aligned}
$$

Thus, we have

$\left(U_{m}\right)_{m \geq 0}$ converges to $U_{0}+\frac{4 \pi \alpha^{2} U_{0}^{\prime}}{N\left(4-\alpha^{2}\right)}$ for all $\theta \in[0, \alpha \pi], \quad N \in \mathbb{N}^{*}$.

Finally, we can deduce the following result:

Proposition 9. Let $T: H_{0}^{1}[a, b] \rightarrow H_{0}^{1}[a, b]$ be a $k$-contraction and $\left(\psi_{n}\right)_{n \geq 0}$ be a sequence of functions defined by

$$
\begin{gathered}
\psi_{n+1}:=T \psi_{n} \text { so that, } \\
\left(T \psi_{n}\right)^{\prime}:=k \psi_{n}^{\prime}, \quad n \geq 0 .
\end{gathered}
$$

If $\left(\psi_{n}\right)_{n \geq 0}$ verifies this recurrence relation

$$
\psi_{n+1}=\psi_{n-1}+\varepsilon\left(T \psi_{n}\right)^{\prime}, \text { with } \varepsilon>0 \text { and } n \in \mathbb{N} \text {. }
$$

Then,

$$
\left(\psi_{n}\right)_{n \geq 0} \text { converges to } \psi_{0}+C(k, \varepsilon) \psi_{0}^{\prime}
$$

$C$ a constant depending on $k$ and $\varepsilon$.
Proof of Proposition 10. The Picard theorem on the convergence of $\left.\left(\psi_{n}\right)_{n \geq 0}\right)$.

We can write

$$
\psi_{n+1}=\psi_{0}+\varepsilon\left(T \psi_{1}+T \psi_{3}+T \psi_{5}+\cdots+T \psi_{n}\right)^{\prime} n \in \mathbb{N}
$$

So,

$$
\psi_{n+1}=\psi_{0}+k^{2} \varepsilon\left(\psi_{0} \frac{1-k^{4 n-4}}{1-k^{2}}\right), \quad n \in \mathbb{N} .
$$

This yields the result.

Further, we set $U_{0}=c\left(e^{i \theta^{2}(\alpha \pi-\theta)^{2}}-1\right), c \in \mathbb{C}$. We have

$$
\begin{aligned}
\psi^{1}(\theta)= & c e^{i \theta^{2}(\alpha \pi-\theta)^{2}}\left[1+\frac{4 \pi \alpha^{2} \theta(\alpha \pi-\theta)(\alpha \pi-2 \theta) i}{N\left(4-\alpha^{2}\right)}\right] \\
& -c \text {, with } c \in \mathbb{C} \text { and } N \in \mathbb{N}^{*} .
\end{aligned}
$$

It is evident that $\psi^{1}$ does not depend on $n$.

Therefore, according to (20) and (21), the map $\Phi^{1}$ is then defined by

$$
\begin{aligned}
\Phi^{1}\left(e^{i \theta}\right)= & \theta+c e^{i \theta^{2}(\alpha \pi-\theta)^{2}}\left[1+\frac{4 \pi \alpha^{2} \theta(\alpha \pi-\theta)(\alpha \pi-2 \theta) i}{N\left(4-\alpha^{2}\right)}\right] \\
& -c, \text { for all } \theta \in[0 ; \alpha \pi] .
\end{aligned}
$$

with $c \in \mathbb{C}$ and $N \in \mathbb{N}^{*}$. The coefficient $a$ is determined using the available Cauchy data and eigenvalues.

For all $z_{0} \in D_{1},\left(\Phi^{1}\right)^{\prime}\left(z_{0}\right) \neq 0$ so $\Phi^{1}$ is holomorphic.

In the following argument, we pose $\Phi=\Phi_{n, k}^{1}, \quad c=a_{n, k}$ because they are determined by eigenvalues.

$\Phi_{n, k}^{1}$ is analytic, continuous on $\bar{D} 1$; it realizes a conformal mapping from $C_{1}$ onto $\partial \Omega$, according to Theorem 1.4 .5 (see [2] page 42). Further, it realizes a conformal mapping (univalent) from the domain $D$ onto the domain $\Omega$.

Hence, for all $0<\alpha<2$, and all $N \in \mathbb{N}^{*}$, there exists an univalent holomorphic function $\Phi_{n, k}^{1}$, which realizes a conformal mapping from the domain $D_{1}$ onto the domain $\Omega$, which is a solution of problem (19), defined by

$$
\begin{aligned}
\Phi_{n, k}^{1}\left(R e^{i \theta}\right)= & \theta+a_{n, k} R e^{i \theta^{2}(\alpha \pi-\theta)^{2}}\left[1+\frac{4 \pi \alpha^{2} \theta(\alpha \pi-\theta)(\alpha \pi-2 \theta) i}{N\left(4-\alpha^{2}\right)}\right] \\
& -a_{n, k} .
\end{aligned}
$$

For all $\theta \in[0, \alpha \pi]$ with $N \in \mathbb{N}^{*}, a_{n, k}, \in \mathbb{C}, 0 \leq R \leq 1 . a_{n, k}$ is determined using the available Cauchy data and eigenvalues.

Proposition 11. Consider the problem (1) - (2) - (3) where $\partial \Omega=\Gamma_{0} o \Gamma$, with $\Gamma$ the unknown part. 
$\partial \Omega$ is a spiral defined on $[0,2 \pi]$ by the following equations:

$$
\left\{\begin{array}{l}
X=\theta-a_{1}+\left(a_{1}-a_{2} h_{1}(\theta)\right) \cos t-\left(a_{1} h_{1}(\theta)+a_{2}\right) \sin t \\
Y=-a_{2}+\left(a_{1} h_{1}(\theta)+a_{2}\right) \cos t+\left(a_{1}-a_{2} h_{1}(\theta)\right) \sin t
\end{array}\right.
$$

with $a_{n, k} \in \mathbb{C}, a_{1}=\left|a_{n, k}\right| \cos \left(\arg \left(a_{n, k}\right)\right), a_{2}=\left|a_{n, k}\right| \sin \left(\arg \left(a_{n, k}\right)\right)$,

$$
h_{1}(\theta)=\frac{4 \pi \alpha^{2} \theta(\alpha \pi-\theta)(\alpha \pi-2 \theta)}{N\left(4-\alpha^{2}\right)} \text { and } t=\theta^{2}(\alpha \pi-\theta)^{2} \text {. }
$$

Then, $\Gamma$ is a part of the spiral, $\partial \Omega$, image of $[0, \alpha \pi]$ under

$\Phi_{n, k}^{1}\left(R e^{i \theta}\right)=\theta+a_{n, k} R e^{i \theta^{2}(\alpha \pi-\theta)^{2}}\left[1+\frac{4 \pi \alpha^{2} \theta(\alpha \pi-\theta)(\alpha \pi-2 \theta) i}{N\left(4-\alpha^{2}\right)}\right]-a_{n, k}$

defined for all $n \geq 1$ and $k \geq 0$.

Proof of the Proposition 12. It suffices to determine the integral and decimal parts of $\Phi_{n, k}^{1}$.
3.1.7. Numerical Simulations. We determine the approximate shape of the unknown part of the boundary corresponding to the two first eigenvalues.

Let $\Phi_{\mathrm{n}, \mathrm{k}}^{1}$ be the map defined in (78).

We put $g(x, y)=0$ and $h(x, y)=-1-x^{2}-y^{2}$, where $h$ is defined to be negative.

First, we determine $a_{n, k}$ of $\Phi_{n, k}^{1}$ corresponding to the first and second eigenvalues, after approximating the corresponding shapes of $\Gamma_{2}$. Using the boundary values, we have previously established,

$$
\begin{gathered}
\Phi_{n}^{\prime}\left(e^{i \theta}\right) \approx \frac{\sum_{k=0}^{+\infty} p_{k, n}\left(\lambda_{k, n}\right) e^{i k \theta}}{h o \Phi_{n}\left(e^{i \theta}\right)}, \forall n \geq 1, \\
u\left(\Phi_{k, n}\left(e^{i \theta}\right)\right)=0, \text { for } \widehat{u} \text { defined in }(16) .
\end{gathered}
$$

The coefficients $a_{1}$ and $a_{2}$ are determined based on the Cauchy data corresponding to each eigenvalue of the Laplacian operator. Hence, they are obtained by

$$
\Phi_{1}^{\prime}\left(e^{i \theta}\right) \approx \frac{\sum_{k=0}^{+\infty}\left(\left((2 n+k-1)^{2} \pi(-1)^{n} \sqrt{2 \pi}\right) / \sqrt{(2 n+k-1)^{2} \pi^{2}-2\left(1+(-1)^{k}\right)}\right) e^{i k \theta}}{h o \Phi_{n}\left(e^{i \theta}\right)}
$$
$\theta=0$ :

Therefore, the calculation yields that corresponding to

$$
\left\{\begin{array}{l}
a_{1}=0 \\
a_{2}=\frac{N\left(4-\alpha^{2}\right)}{4 \alpha^{4} \pi^{3}}\left(1+\sum_{k=0}^{+\infty} \frac{(2 n+k-1)^{2} \pi(-1)^{n} \sqrt{2 \pi}}{\sqrt{(2 n+k-1)^{2} \pi^{2}-2\left(1+(-1)^{k}\right)}}\right) .
\end{array}\right.
$$

\subsubsection{Conformal Map $\Phi_{0,1}$ Corresponding to First Eigenvalue}

$\lambda_{0,1}$. Corresponding to the first eigenvalue $\lambda_{0,1}$, we have

$$
\begin{aligned}
& a_{2}=\frac{N\left(4-\alpha^{2}\right)}{4 \alpha^{4} \pi^{3}}\left(1-\frac{\pi \sqrt{2 \pi}}{\sqrt{\pi^{2}-4}}\right), \\
& \left\{\begin{array}{l}
X=\theta-\frac{N\left(4-\alpha^{2}\right)}{4 \alpha^{4} \pi^{3}}\left(1-\frac{\pi \sqrt{2 \pi}}{\sqrt{\pi^{2}-4}}\right)\left(\frac{4 \pi \alpha^{2} \theta(\alpha \pi-\theta)(\alpha \pi-2 \theta)}{N\left(4-\alpha^{2}\right)} \cos t+\sin t\right), \\
Y=\frac{N\left(4-\alpha^{2}\right)}{4 \alpha^{4} \pi^{3}}\left(1-\frac{\pi \sqrt{2 \pi}}{\sqrt{\pi^{2}-4}}\right)\left(-1+\cos t-\frac{4 \pi \alpha^{2} \theta(\alpha \pi-\theta)(\alpha \pi-2 \theta)}{N\left(4-\alpha^{2}\right)} \sin t\right) .
\end{array}\right.
\end{aligned}
$$




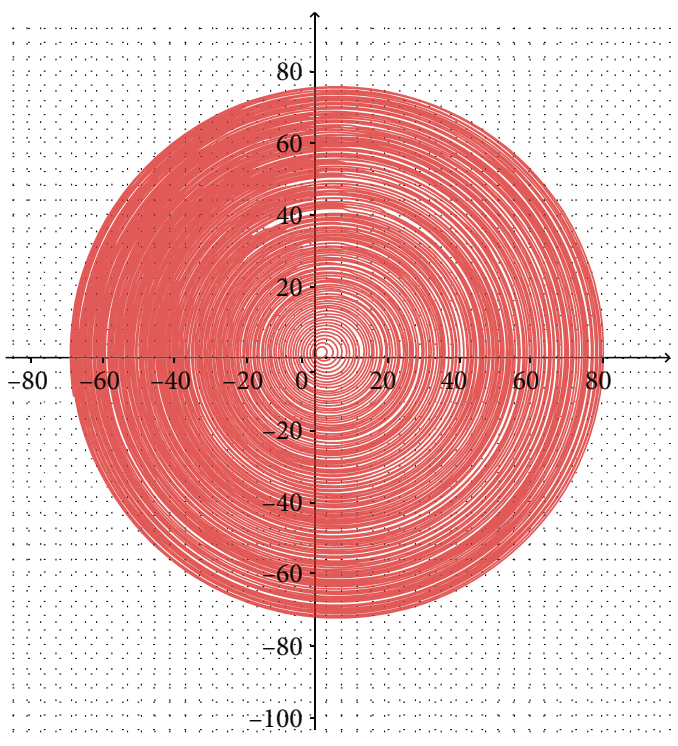

(a)

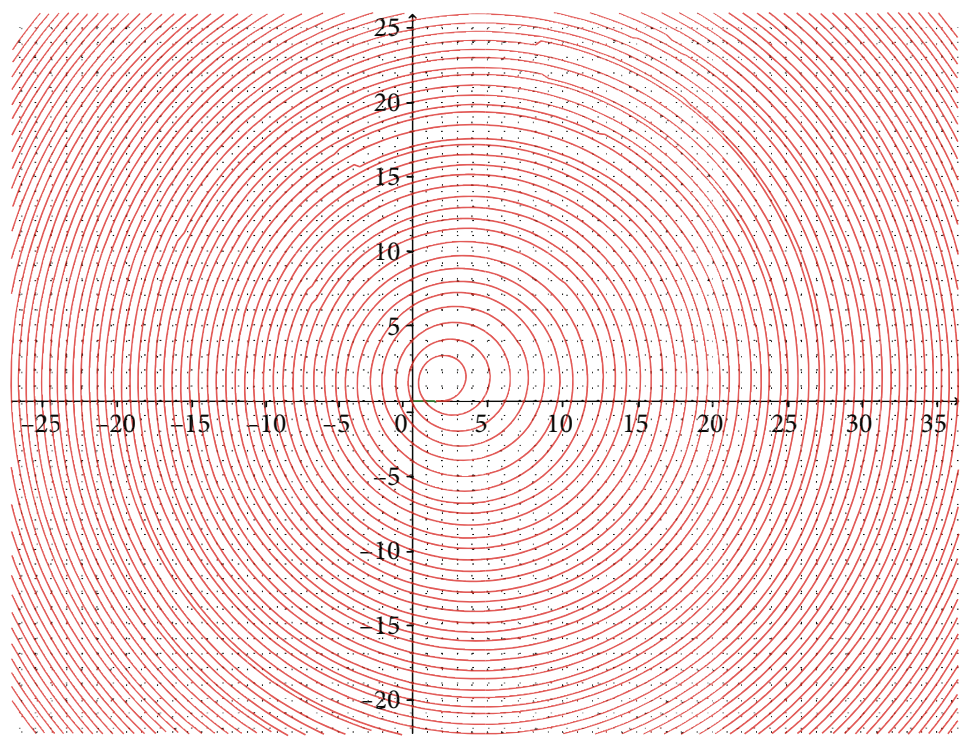

(b)

FIgURe 1: N5 Alpha0.5.

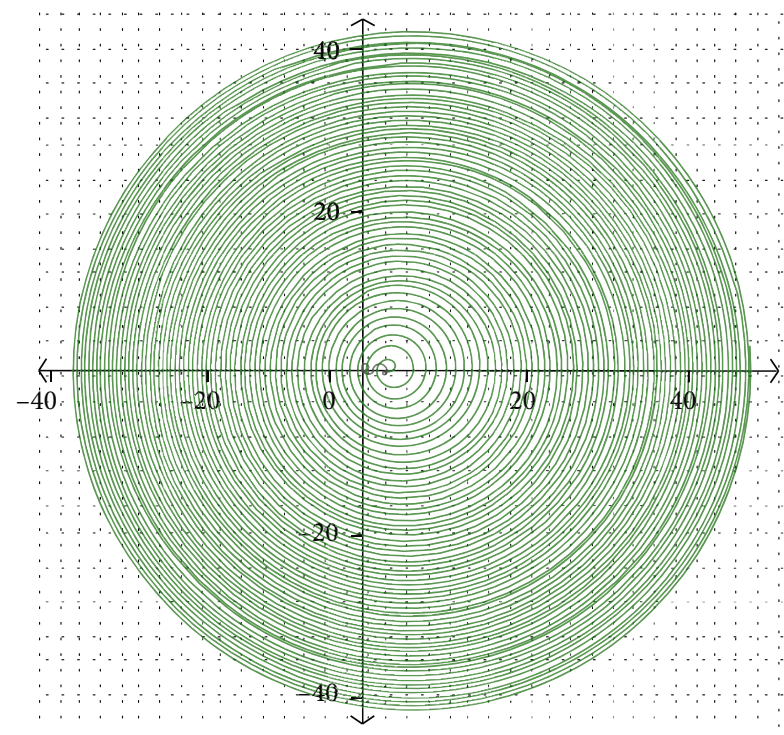

(a)

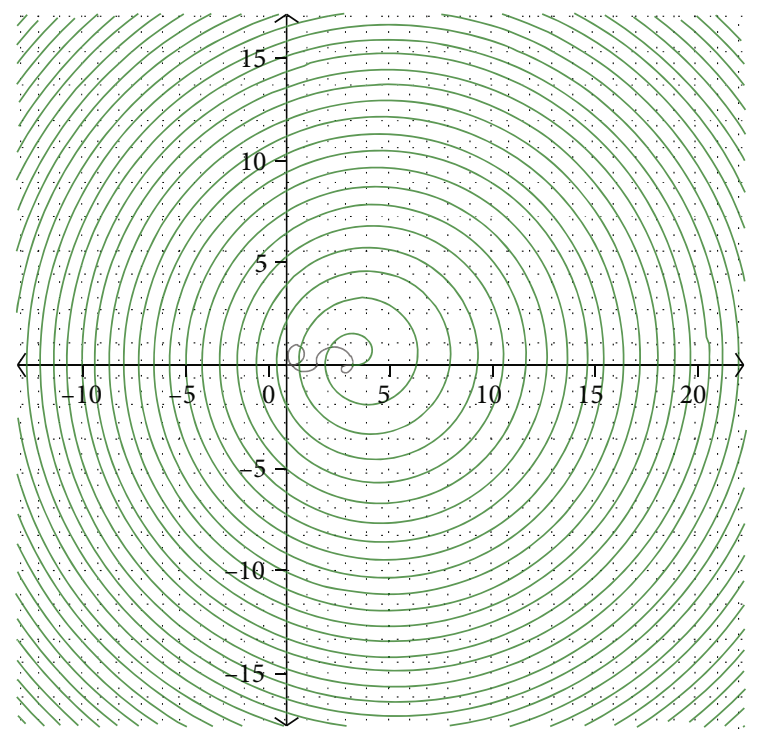

(b)

FIgURe 2: N5 Alpha1.

Therefore, we obtain the result for

(1) $N=5$ and $\alpha=0.5$

Figure 1 is obtained by applying $N=5$ and $\alpha=0.5$ in (86). Figure 1(a) shows the shapes of $\Gamma$ and $\Gamma_{0}$ in an orthogonal mark with graphic unit $20 \mathrm{~cm}$, and Figure 1(b) shows these same shapes in an orthogonal mark with graphic unit $5 \mathrm{~cm}$. It is this right part which gives us the enlargement of shapes of $\Gamma$ and $\Gamma_{0}$.

(2) $N=5$ and $\alpha=1$
Figure 2 is obtained by applying $N=5$ and $\alpha=1$ in (86). Figure 2(a) shows the shapes of $\Gamma$ and $\Gamma_{0}$ in an orthogonal mark with graphic unit $20 \mathrm{~cm}$, and Figure 2(b) shows these same shapes in an orthogonal mark with graphic unit $5 \mathrm{~cm}$. It is this right part which gives us also the enlargement of shapes of $\Gamma$ and $\Gamma_{0}$.

It is to be noted that, in each case $(\alpha=0.5$ or $\alpha=1), \Gamma_{0}$ is sinusoïdal, $\Gamma$ is a part of spiral, and $\partial \Omega$ converges to a finite limit. Therefore, $\partial \Omega$ is asymptotic to a circle and $\Omega$ is disk shaped. 


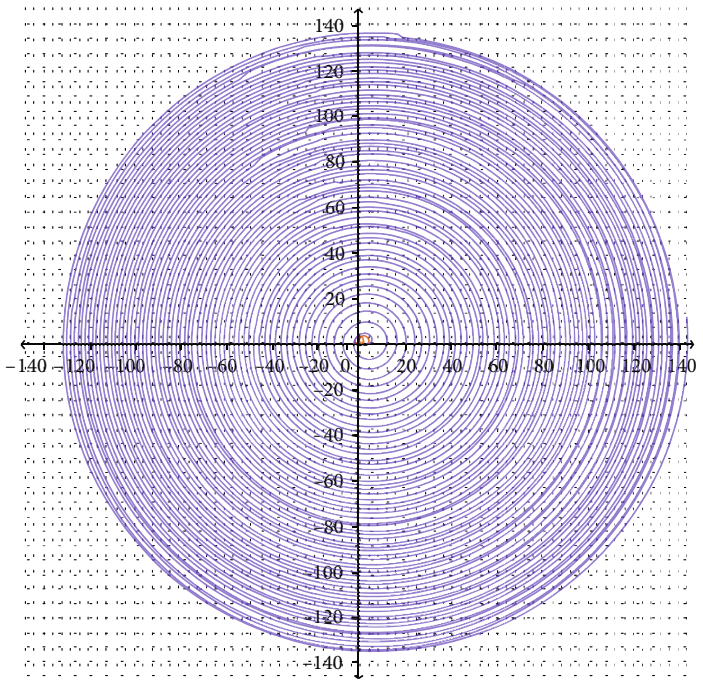

(a)

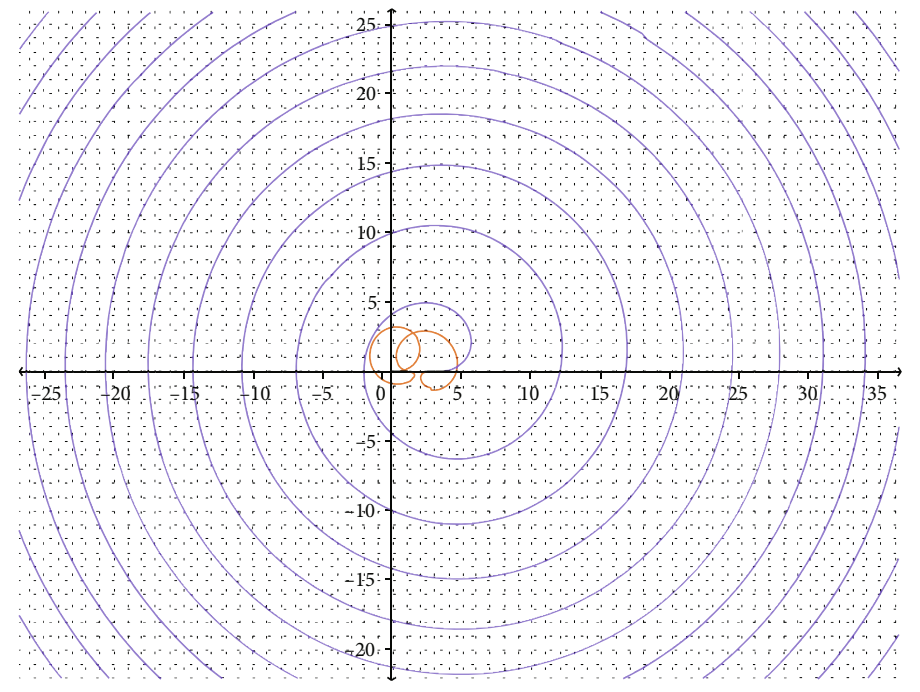

(b)

Figure 3: N5 Alpha1.

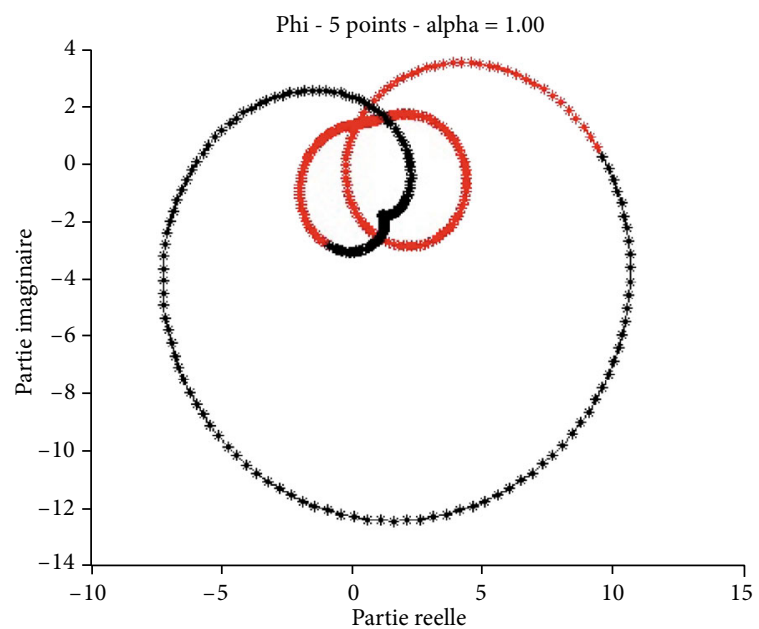

(a)

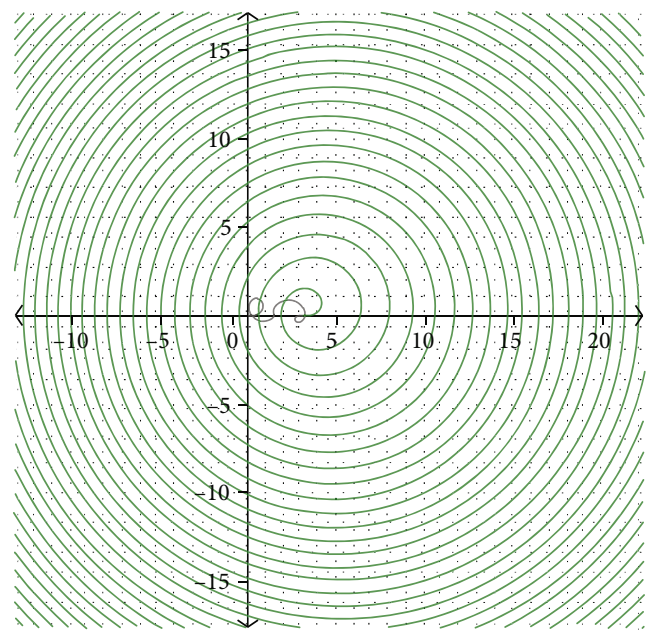

(b)

FIgUre 4: Results for $\Gamma$ of the previous study in 2014 black in (a). Results for $\Gamma$ of the present study green to (b).

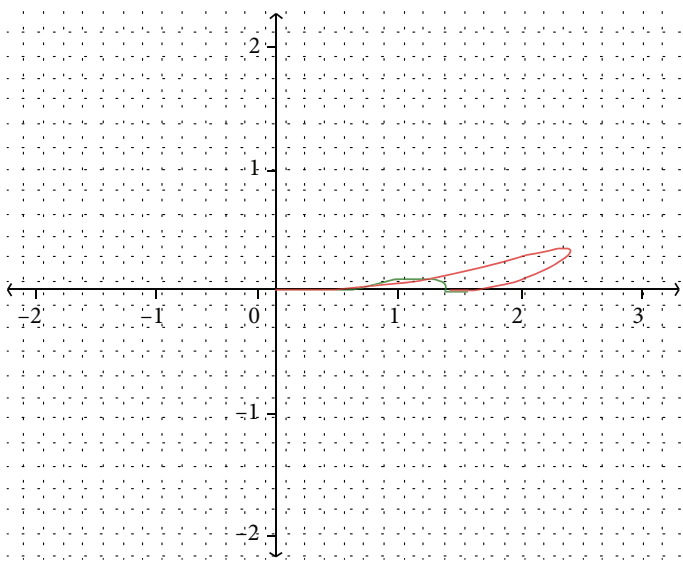

(a)

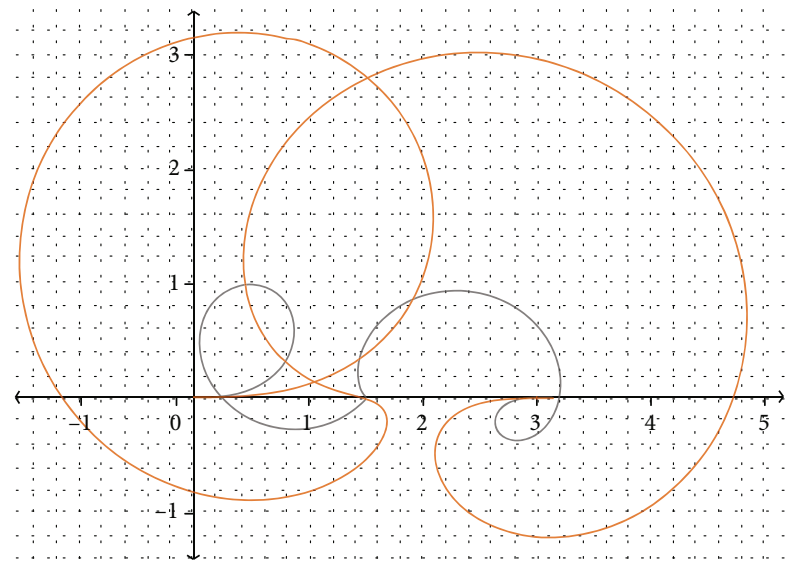

(b)

FIgURE 5: Variations of $\Gamma_{0}$ with respect to $\lambda_{n, k}$ 
3.1.9. Conformal Map $\Phi_{1,1}$ Corresponding to the Second Eigenvalue $\lambda_{1,1}$. Corresponding to the second eigenvalue $\lambda_{1,1}$, we have

$$
\begin{gathered}
a_{2}=\frac{N\left(4-\alpha^{2}\right)}{4 \alpha^{4} \pi^{3}}\left(1-\frac{\pi \sqrt{2 \pi}}{\sqrt{\pi^{2}-4}}-2 \sqrt{2 \pi}\right), \\
X=\theta-\frac{N\left(4-\alpha^{2}\right)}{4 \alpha^{4} \pi^{3}}\left(1-\frac{\pi \sqrt{2 \pi}}{\sqrt{\pi^{2}-4}}-2 \sqrt{2 \pi}\right)\left(\frac{4 \pi \alpha^{2} \theta(\alpha \pi-\theta)(\alpha \pi-2 \theta)}{N\left(4-\alpha^{2}\right)} \cos t+\sin t\right), \\
Y=\frac{N\left(4-\alpha^{2}\right)}{4 \alpha^{4} \pi^{3}}\left(1-\frac{\pi \sqrt{2 \pi}}{\sqrt{\pi^{2}-4}}-2 \sqrt{2 \pi}\right)\left(-1+\cos t-\frac{4 \pi \alpha^{2} \theta(\alpha \pi-\theta)(\alpha \pi-2 \theta)}{N\left(4-\alpha^{2}\right)} \sin t\right) .
\end{gathered}
$$

Thus, we conclude the result for $N=5$ and $\alpha=1$.

Figure 3 is obtained by applying $N=5$ and $\alpha=1$ in (87). Here too, Figure 3(a) shows the shapes of $\Gamma$ and $\Gamma_{0}$ in an orthogonal mark with graphic unit $20 \mathrm{~cm}$, and Figure 3(b) shows these same shapes in an orthogonal mark with graphic unit $5 \mathrm{~cm}$. Figure 3(a) gives us also the enlargement of shapes of $\Gamma$ and $\Gamma_{0}$.

Here too, $\Gamma_{0}$ is sinusoïdal, $\Gamma$ is a part of spiral, and $\partial \Omega$ converges to a finite limit.

3.1.10. Comparison Results for $N=5$ and $\alpha=1$. The result for the first eigenvalue obtained via the minimization functional method for $N=5$ and $\alpha=1$ in our previous study, and the result obtained using the fixed-point method for the same data has been depicted in the following diagram.

In Figure 4, we can observe that

(i) $\Gamma_{0}$ is sinusoïdal in both these studies

(ii) $\Gamma$ is a part of spiral in the present study and is a part of spiral with a cusp, in the study in 2014

(iii) Both methods yielded a spiral shape for $\partial \Omega$

3.1.11. Variations of $\Gamma_{0}$ with respect to $\lambda_{n, k}$. The following diagram allows us to compare the shapes of $\Gamma_{0}$ corresponding the results in the first and the second study for the first eigenvalues.

In Figure 5, we obtain results of the variations of the shape of $\Gamma_{0}$ with respect to $\lambda_{n, k}$. Figure 5(a) shows the variation of the shapes of $\Gamma_{0}$ by applying $N=5$ and $\alpha=0.5$ in (86) and in (87), i.e., for the first and second eigenvalues. Figure 5(b) shows the variation of the shapes of $\Gamma_{0}$ by applying $N=5$ and $\alpha=1$ in (86) and in (87), i.e., for the first and the second eigenvalue.

3.2. Results. All previous figures are obtained using "GeoGebra Geometry."

Figures 1(b), 2(b), and 3(b) give us the enlargement of shapes of $\Gamma$ and $\Gamma_{0}$ found, and they allow us to see their exact shapes. Also, these exact shapes allow us to make a good interpretation of the results.
So, the simulations yield the following results:

(1) The shape of $\Gamma_{0}$ is a spiral. When the value of $\alpha$ increases, its spiral shape is accentuated, and it is constituted of identical connected parts. Further, when the eigenvalue increases, the length of $\Gamma_{0}$ is observed to increase accompanied by a change in its spiral shape (see Figure 5).

(2) However, the shape of $\Gamma$ is a spiral corresponding to all variations of $\alpha$ and the eigenvalues. Its shape does not vary and it converges to a finite limit, which is circular in shape.

(3) Figure 4(a) shows the results of the previous study, which was conducted in 2014, for $N=5$ and $\alpha=1$, and Figure 4(b) shows the results of the present study for $N=5$ and $\alpha=1$. We can observe that the shape of $\partial \Omega$ is spiral in both the results; however, the present study provides a more precise shape of the spiral that is asymptotic to a circle. This shape can justify that $\Omega$ is a disk.

Therefore, the result obtained using the fixed-point method is more precise and further identifies $\Omega$ as a disk.

\section{Conclusion}

In summary, $\Phi_{n, k}$ preserves the spiral shape of $\Gamma$ and its properties but it varies those of $\Gamma_{0}$ based on variations in eigenvalues.

Therefore, $\partial \Omega$ is a spiral, which is asymptotic to a circle and $\Omega$ is identified to be a disk.

This result can be applied to the one-dimensional case, but it is more interesting to study this phenomenon in dimensions three and four.

In an inverse problem with similar conditions, it is more relevant to use conformal mapping and the fixed-point method to yield interesting numerical results. The general case that $\Omega$ is a disk remains an open problem. 


\section{Data Availability}

The data used to support the findings of this study are available from the corresponding author upon request.

\section{Conflicts of Interest}

The author declares that there is no conflict of interest.

\section{Acknowledgments}

We would like to thank Editage (https://www.editage.com) for English language editing services.

\section{References}

[1] R. Kress, "Inverse Dirichlet problem and conformal mapping," Mathematics and Computers in Simulation, vol. 66, no. 4-5, pp. 255-265, 2004.

[2] F. Ndiaye, Etude de problèmes de localisation: Identification de sites et de données, [M.S. thesis], UCAD, 2014.

[3] F. Ndiaye, I. Ly, B. M. Ndiaye, and D. Seck, "Reconstruction method for conformal mapping in two dimensions," AMO Advanced Modeling and Optimization, vol. 16, no. 1, pp. 143$163,2014$.

[4] H. Haddar and R. Kress, "Conformal mappings and inverse boundary value problems," Inverse Problems, vol. 21, no. 3, pp. 935-953, 2005.

[5] I. Akduman and R. Kress, "Electrostatic imaging via conformal mapping," Inverse Problems, vol. 18, no. 6, pp. 1659-1672, 2002.

[6] R. Schinzinger and P. A. Laura, Conformal Mapping, Methods and ApplicationsDover Publications, Inc, Mineola, NY, USA.

[7] J. -P. Demailly, Analyse Numérique et Équations Différentielles, Presse universitaire de Grenoble, 1996.

[8] C. Basdevant, Équations Différentielles, Etude Théorique et Schémas Numériques, 2003-2004. 